\title{
1 Phylogenetic approaches reveal biodiversity threats under climate change
}

2 Carlos E. González-Orozco ${ }^{1,2^{*}}$, Laura J. Pollock ${ }^{3,4,5}$, Andrew H. Thornhill ${ }^{6,7,8}$, Brent D.

3 Mishler $^{6}$, Nunzio Knerr ${ }^{7}$, Shawn W. Laffan ${ }^{8}$, Joseph T. Miller ${ }^{7,9}$, Dan F. Rosauer ${ }^{11}$, Daniel P.

4 Faith $^{12}$, David A. Nipperess ${ }^{13}$, Heini Kujala ${ }^{5}$, Simon Linke ${ }^{14}$, Nathalie Butt ${ }^{15}$, Carsten

5 Külheim $^{11}$, Michael D. Crisp ${ }^{11}$, Bernd Gruber ${ }^{1}$.

6

$7 \quad{ }^{1}$ Institute for Applied Ecology and Collaborative Research Network for Murray-Darling

8 Basin Futures, University of Canberra, ACT 2601, Australia. ${ }^{2}$ Corporación Colombiana de

9 Investigación Agropecuaria, Corpoica, km 17 Vía Puerto López, Meta, Colombia. *e-mail:

10 cegonzalez@corpoica.org.co. ${ }^{3}$ Univ. Grenoble Alpes, Laboratoire d'Écologie Alpine

11 (LECA), F-38000 Grenoble, France. ${ }^{4}$ CNRS, Laboratoire d'Écologie Alpine (LECA), F-

1238000 Grenoble, France. ${ }^{5}$ National Environmental Research Program, School of Biosciences,

13 The University of Melbourne, Melbourne, Victoria 3010, Australia. ${ }^{6}$ University and Jepson

14 Herbaria, and Dept. of Integrative Biology, University of California, Berkeley, CA 94720-

15 2465, USA. ${ }^{7}$ National Research Collections Australia, CSIRO National Facilities and

16 Collections, GPO Box 1600, Canberra, ACT 2601, Australia. ${ }^{8}$ Australian Tropical Herbarium,

17 James Cook University, Cairns QLD 4870, Australia. ${ }^{9}$ Division of Environmental Biology,

18 National Science Foundation, Arlington, Virginia 22230 USA. ${ }^{10}$ Centre for Ecosystem

19 Science, School of Biological, Earth and Environmental Sciences, University of New South

20 Wales, Sydney 2052, Australia. ${ }^{11}$ Research School of Biology, Australian National

21 University, Acton, ACT, 2601, Australia. ${ }^{12}$ The Australian Museum Research Institute, The

22 Australian Museum, Sydney, NSW 2010, Australia. ${ }^{13}$ Department of Biological Sciences,

23 Macquarie University, NSW 2109, Australia. ${ }^{14}$ Australian Rivers Institute, Griffith

24 University, Nathan, Qld, 4111, Australia. ${ }^{15}$ ARC Centre of Excellence for Environmental

25 Decisions and School of Biological Sciences, The University of Queensland, St. Lucia, 4072. 
Predicting the consequences of climate change for biodiversity is critical to conservation efforts $^{1,2,3}$. Extensive range losses have been predicted for thousands of individual species $^{4}$, but less is known about how climate change might impact whole clades ${ }^{1}$ and landscape-scale patterns of biodiversity ${ }^{5}$. Here, we show that climate change scenarios imply significant changes in phylogenetic diversity and phylogenetic endemism at a continental scale in Australia using the hyper-diverse clade of eucalypts. We predict that within the next 60 years the vast majority of species distributions (91\%) across Australia will shrink in size (on average by 51\%) and shift south based on projected suitable climatic space. Geographic areas currently with high phylogenetic diversity and endemism are predicted to change substantially in future climate scenarios.

Approximately $90 \%$ of the current areas with concentrations of paleo-endemism ${ }^{6}$ (i.e. places with old evolutionary diversity) are predicted to disappear or shift their location. These findings show that climate change threatens whole clades of the phylogenetic tree, and can be used to forecast areas of biodiversity losses and continental scale impacts of climate change.

\section{By combining species distribution models (SDMs) with measures of evolutionary} diversity, we can predict how climate change might impact the tree of life in the future ${ }^{1,7,8}$. Preserving the tree of life is increasingly recognised as an important consideration for conservation in Australia; globally, this evolutionary heritage provides a storehouse of unanticipated benefits for future generations, and locally it helps ensure resilient ecosystems and ongoing delivery of their services ${ }^{9,10,11,12}$. The most commonly used metric to quantify the diversity represented by the tree of life is phylogenetic diversity (PD), which represents the shared evolutionary history of species present in a region and is measured as the sum of branch lengths in the phylogenetic tree linking the species present ${ }^{10}$. PD does not include information on rarity of lineages, which is another important concern for conservation 
51 priorities. Phylogenetic endemism (PE) addresses this problem by incorporating the degree of

52 spatial restriction of phylogenetic branches found in an area, relative to all other areas ${ }^{13}$. This

53 useful property means that PE can be used to identify areas that host relatively unique

54 diversity, such as range-restricted "long branch" lineages (i.e., paleoendemics - species or

55 clades with no living close relatives such as the reptile Tuatara, Sphenodon, or the fish

56 Coelacanth, Latimeria).

57 In addition to preserving past evolutionary heritage, protecting areas that hold rapidly diversifying, range-restricted lineages (i.e., neoendemics) is a priority, because those lineages hold the key to future evolutionary potential. Previous studies have considered how climate change will impact the evolutionary heritage represented by $\mathrm{PD}^{1,7,8}$. PD is expected to be correlated with species richness. Therefore we applied a spatial randomization to evaluate departures of PD and PE from expectations ${ }^{6}$. Consequently, our PD-based metrics are decoupled from their corresponding species-based metrics. Here we present the first analysis describing how both the past and future of evolution might be impacted by climate change using model predictions and newly-developed phylogenetic metrics that take into account relative branch lengths.

We explored our approach for a key taxonomic group, Australian eucalypts (genera Eucalyptus, Corymbia, and Angophora). Australian eucalypts are an appropriate biological model because they are a hyper-diverse group ( $>800$ species) that dominate forest canopies and ecosystems across much of the continent. Eucalypts are mostly endemic to Australia and

71 have a broad biogeographical diversity ${ }^{14}$. They are of global interest due to their widespread

72 use in forestry ${ }^{15}$. They form a monophyletic lineage within the Myrtaceae family ${ }^{16,17,18,19}$,

73 including the few eucalypt species that occur outside of Australia. Here we examine the

74 expected shift in the geographic ranges of 657 species of eucalypt across Australia, 75 comparing present ranges to those under forecast climate change scenarios for the years 2025, 
762045,2065 and 2085. We used SDMs to predict changes in geographic ranges and the

77 direction in which ranges are likely to shift under future climate scenarios. SDMs have

78 limitations when predicting responses to climate change ${ }^{20}$ in species (e.g., climatic

79 adaptability) and communities (e.g., species interactions). We accounted for some of the

80 well-known weaknesses of SDMs by explicitly considering spatial bias and over-fitting, and

81 comparing models with and without dispersal. The dispersal and non-dispersal scenarios

82 produced very similar patterns, so we report only the results from the dispersal scenario.

83 Our study's novel findings regarding effects of climate change on phylogenetic

84 diversity are grounded in our descriptions of impacts at the species level that corroborate

85 previous findings. Eucalypt species are known to be threatened by climate change in

86 Australia with previous results suggesting 53\% of all Eucalyptus species predicted to be

87 outside of their current climate conditions by 2070 under a $3^{\circ} \mathrm{C}$ warming scenario ${ }^{21}$. More

88 recent SDM modelling for eucalypts suggests species ranges across the continent will

contract and shift in both lateral and poleward directions, and the central desert areas will be

90 particularly affected ${ }^{22}$. Our models predicted that by 2085 , the current geographic range of

$9191 \%$ of the 657 eucalypt species will shrink by an average of $51 \%$, with $2.4 \%$ of species

92 predicted to become extinct because their climate space will disappear entirely. Relatively

93 few species (9\%) are predicted to expand their geographic range (Fig. 1; see also SI_2 for

94 range size, \% change, type of shift, shift magnitude and shift direction; and SI_4 for maps for

95 each of the 657 species).

96 To understand how these losses in species will manifest as changes in evolutionary

97 diversity across the landscape, we calculated present and future $\mathrm{PD}^{10}$ and $\mathrm{PE}^{13}$ for all grid

98 cells $(\sim 25 \mathrm{~km} \times 25 \mathrm{~km})$. These analyses predict that on average $2 \%$ of PD (decline in PD grid

99 cells) will be lost across Australia by 2085 (Extended Data Fig. 1). Losses will occur in

100 places recognised as national ${ }^{14}$ and global biodiversity hotspots ${ }^{23}$. Additionally, we found that 
the rate of turnover of PD increases over time, with a higher proportion of major changes occurring in southern Australia (Extended Data Fig. 9). Specifically, Mediterranean, temperate, and grassland ecosystems will face the greatest proportional change in clades present over time. This trend is accompanied by an increasing homogenization over the landscape. The average rate of phylogenetic turnover between all pairs of cells within a given year will decrease from 0.58 in the present to 0.50 in 2085 (Extended Data Table 1), suggesting an increase in average spatial homogeneity of phylogenetic composition (or phylogenetic homogenization) in the future. This spatial homogenization of PD has been documented previously for diverse groups in Europe ${ }^{1}$, and is suggested by past and current climate-driven plant extinctions for Thoreau's woods in the US and some temperate tree floras in Europe $\mathrm{e}^{24,25}$. Here, for the first time, we show the potential effects of climate-change leading to PD loss in the future for a significant biological group of the southern hemisphere. In contrast, $\mathrm{PE}$ is predicted to increase spatially in some of the areas of key diversity hotspots. In general, species endemism and PE are measures of rarity, and therefore, endemism tends to increase in the areas that hold the remaining portions of species ranges. The increase in PE is a result of lineages becoming more range-restricted. For example, in southwestern Western Australia, an internationally recognized biodiversity hotspot, species are predicted to retract toward the coast causing some coastal cells to have high PE in the future (Extended Data Fig. 1e). Predicting the location of high PE areas in the future is important from a conservation standpoint because these locations are likely to be the sites of concentrations of rare elements of diversity in the future, and, therefore, will be strategic areas for preventing further PD losses.

Two derived metrics, relative phylogenetic diversity (RPD) and relative phylogenetic endemism (RPE) ${ }^{6}$, can be used to better understand anticipated impacts on unusually long or short branches. These metrics reveal information about the underlying evolutionary processes 
126 that we seek to understand and conserve ${ }^{6}$ and also because they store old and highly unique

127 lineages that, once lost, will significantly reduce the breadth of biodiversity. Sites with many very long branches are important repositories of unique evolutionary history and trait diversity $^{26}$; sites with many short branches might indicate places with the potential for generating evolutionary adaptation and speciation in the future.

The RPD and RPE metrics reveal these patterns through an assessment that asks whether the contribution of unusually long or short branches to PD or PE in a grid cell is greater or less than expected by chance. RPD (or RPE) is the ratio of PD (or PE) measured using the set of species in that grid cell on the observed tree of the 657 eucalypt species to that measured on a comparison tree with the same topology but with all branches adjusted to be of equal (average) length ${ }^{6}$, with significant large or small ratios determined by a spatial randomization test (see Methods for details of randomization test) ${ }^{27}$. We find both areas of significantly high and significantly low RPD are predicted to decline in size $(\sim 10 \%)$ by 2085 (Extended Data Fig. 2), indicating that unusually long- and short-branch lineages will disappear within sites.

Endemism can result either from younger lineages that have yet to expand their range or from older lineages that are relicts of a previously broader distribution. RPE allows the identification of centres of paleo-endemism (range-restricted long branches), centres of neoendemism (range-restricted short branches), or both (centres of mixed endemism, termed "super endemism"), through a recently-developed method called CANAPE (Categorical Analysis of Neo- And Paleo-Endemism; see methods $)^{6}$. We find that the current areas of paleo-, neo-, and super-endemism are predicted to significantly shrink $(\sim 50 \%)$ in size by 2085 (Fig. 2; Extended Data Fig. 3a-e). This suggests that current areas of significant phylogenetic endemism may decline as lineages either go extinct or move to different locations (Figs. 2 and 3) with climate change. 
Areas of paleo-endemism are particularly impacted; $\geq 90 \%$ of the current areas with

significantly high paleo-endemism will disappear or shift to new places by 2085 (Fig. 2 ellipses). Most of the large loss of paleo-endemism is due to shrinking geographic range of lineages (Extended Data Fig. 4). This large loss of paleo-endemism is a loss in the number of grid cells falling into that category. Shrinking ranges can increase the paleo-endemism of a cell that retains those long branches, but overall decreases of paleo-endemism areas reflects the loss of those branches from areas. For example, between the present and 2085, 64.6\% of species' geographic ranges within current paleo-endemism areas will shrink (Extended Data Fig. 4), thus impacting their contribution to overall PE of these areas. There are currently 73 grid cells with significant eucalypt paleo-endemism in Australia, containing 240 species. By 2085, 52 of these species are predicted to go extinct or migrate elsewhere (Fig. 3).

Consequently, most of these grid cells will cease to be areas of paleo-endemism by 2085 .

Declines of paleo-endemic areas tended to be even larger in the no-dispersal scenario, which otherwise gave similar results to the dispersal scenario reported in the text (Extended Data Fig. $3 \mathrm{f}-\mathrm{j}$ ). These results show that areas of paleo-endemism are not necessarily places of long-term stability, but instead may be highly threatened and mobile.

We also found that, in a few cases, new areas of paleo-endemism (Fig. 2, squares) are predicted to form. The main reason that paleo-endemic sites appear is because current widespread long-branch lineages become more range-restricted under climate change. These predicted new centres of paleo-endemism will be important for future conservation because they represent predicted areas that have the potential to harbour rare long branches. Lineages are also predicted to disappear from places where new concentrations of paleo-endemism will form (see the square in central Australia and the larger rectangle in the far top-left in Fig. 2b). These sites currently contain 70 and 57 species and we predict that 
$17523.3 \%$ and $7 \%$ of those species, respectively, will be locally extinct by 2085 (Fig. 2;

176 Extended Data Fig. 5c, d).

177 Overall, the extinction of whole lineages is likely to occur as a result of severe

178 reductions of climatic space (Extended Data Fig. 5a-d; and species list in SI_1, Tables 1-4).

179 The effect on the phylogeny was not evenly distributed as has been previously reported in

180 some cases $^{24}$, and we identified potential extinction of five species of Eucalyptus in

181 Southwest Australia and three of Corymbia in southeast Queensland. This could be

considered as an early sign of phylogenetic extirpation ${ }^{25}$ or extinction of lineages of eucalypts 183 (Fig. 3).

Few studies have addressed the impact of climate change on $\mathrm{PD}^{28}$ and ours is the first to take the additional step of showing how climate change might affect PE including centres of paleo- (old) and neo- (young) -endemism across a continental-scale landscape. Our approach can be considered as an early warning system for forecasting biodiversity loss across the landscape, critically expressed as loss of evolutionary heritage and evolutionary potential. Here we show that the contraction of lineage ranges expected with climate change with substantial losses to biodiversity with climate change, our approach allows the identification of areas that are key to preventing further biodiversity loss. realized, would lead to a shrinkage of current areas of high phylogenetic endemism for the species group along all the southwest, southeast, and southern coastal regions of Australia. very important refugium, while Victoria is more prone to losing areas of neo-endemism. 
200 Current hotspots of paleo-endemism in southeast Queensland, northern coastal NSW, and

201 central Australia are also predicted to be impacted severely. Conversely, new concentrations

202 of paleo-endemism are predicted to occur in the Kimberley and northern coastal region

203 demonstrating the potential of our methods to identify key areas that might be important

204 refugia in the future.

205

206

References

207

1. Thuiller, W. et al. Consequences of climate change on the tree of life in Europe. Nature 470, 531-534 (2011).

2. Araujo, M.B., Thuiller, W. \& Pearson, R.G. Climate warming and the decline of amphibians and reptiles in Europe. Journal of Biogeography 33, 1712-1728 (2006).

3. Purvis, A. \& Hector, A. Getting the measure of biodiversity. Nature 405, 212-219 (2000).

4. Hof, C., Araujo, M.B., Jetz, W. \& Rahbek, C. Additive threats from pathogens, climate and land-use change for global amphibian diversity. Nature 480, 22-29 (2011).

5. Warren, R. et al. Quantifying the benefit of early climate change mitigation in avoiding biodiversity loss. Nature Climate Change 3, 678-682 (2013).

6. Mishler, B.D. et al. Phylogenetic measures of biodiversity and neo- and paleo-endemism in Australian Acacia. Nature Communications 5, 4473 (2014).

7. Faith, D.P. \& Richards, Z.T. Climate change impacts on the tree of life: changes in Phylogenetic Diversity illustrated for Acropoda corals. Biology 1, 906-932 (2012).

8. Loyola, R.D. et al. Clade-specific consequences of climate change to amphibians in Atlantic Forest protected areas. Ecography 37, 65-72 (2014).

223 9. Faith, D.P. \& Pollock, L.J. (2014) Phylogenetic diversity and the sustainable use of 
biodiversity. pp 35-52. In: Applied ecology and human dimensions in biological conservation (ed. L.M Verdade et al.) Springer.

10. Faith, D.P. Conservation evaluation and phylogenetic diversity. Biological Conservation 61, 1-10 (1992).

11. Faith, D.P. et al. Evosystem Services: an evolutionary perspective on the links between biodiversity and human-well-being. Current Opinion in Environmental Sustainability 2, 66-74 (2010).

12. Cadotte, M.W. et al. Phylogenetic diversity metrics for ecological communities: integrating species richness, abundance and evolutionary history. Ecology Letters 13, 96-105 (2010).

13. Rosauer, D., Laffan, S.W., Crisp, M.D., Donnellan, S.C. \& Cook, L.G. Phylogenetic endemism: a new approach for identifying geographical concentrations of evolutionary history. Molecular Ecology 18, 4061-4072 (2009).

14. González-Orozco, C.E., Thornhill, A.H., Knerr, N., Laffan, S. \& Miller, J.T. Biogeographical regions and phytogeography of the eucalypts. Diversity \& Distributions 20, 46-58 (2014).

15. Wilson P.G. (2011) Myrtaceae. The Families and Genera of Vascular Plants. Volume X. Flowering Plants Eudicots: Sapindales, Cucurbitales, Myrtaceae (ed. by K. Kubitzki), pp. 212-271. Springer-Verlag, Heidelberg.

16. Thornhill, A.H., Ho S.Y.W., Külheim C. \& Crisp M.D. Interpreting the modern distribution of Myrtaceae using a dated molecular phylogeny. Molecular Phylogenetics and Evolution 93, 29-43 (2015).

17. Thornhill, A.H., Popple L.W., Carter R.J., Ho S.Y.W. \& Crisp M.D. Are pollen fossils useful for calibrating relaxed molecular clock dating of phylogenies? A comparative study using Myrtaceae. Molecular Phylogenetics and Evolution 63, 15-27 (2012). 
18. Crisp, M.D., Burrows G.E., Cook L.G., Thornhill A.H. \& Bowman D.M.J.S. Flammable biomes dominated by eucalypts originated at the Cretaceous-Palaeogene boundary. Nature Communications 2, 1-8 (2011).

19. Bayly M.J., et al. Chloroplast genome analysis of Australian eucalypts-Eucalyptus, Corymbia, Angophora, Allosyncarpia and Stockwellia (Myrtaceae). Molecular Phylogenetics and Evolution 69, 704-16 (2013).

20. Booth, T.H. et al. Native forest and climate change: Lessons from eucalypts. Forest Ecology and Management 347, 18-29 (2015).

21. Hughes, L., Cawsey, E.M. \& Westoby, M. Climatic range sizes of Eucalyptus species in relation to future climate change. Global Ecology and Biogeography Letters 5, 23-29 (1996).

22. Butt, N., Pollock, L.J. \& McAlpine, C.A. Eucalypts face increasing climate stress. Ecology and Evolution 3, 5011-5022 (2013).

23. Myers, N., Mittermeier, R.A., Mittermeier, C.G., da Fonseca, G.A.B. \& Kent, J. Biodiversity hotspots for conservation priorities. Nature 403, 853-858 (2000).

24. Eiserhardt, W.L., Borchsenius, F., Plum, C.M., Ordoñez, A. \& Svening, J-C. Climatedriven extinctions shape the phylogenetic structure of temperate tree floras. Ecology Letters 18, 263-272 (2015).

25. Willis, C.G. et al. Phylogenetic patterns of species loss in Thoreau's woods are driven by climate change. Proc. Natl Acad. Sci. USA 105, 17029-17033 (2008).

26. Forest, F et al. Preserving the evolutionary potential of floras in biodiversity hotspots. Nature 445, 757-760 (2007).

27. Thornhill, A. H et al. Continental-scale spatial phylogenetics of Australian angiosperms provides insights into ecology, evolution and conservation. Journal of Biogeography. doi/10.1111/jbi.12797 (2016). 
274 28. Zhang, J., Nielsen, S.E., Stolar, J., Chen, Y \& Thuiller, W. Gains and losses of plant

275 species and phylogenetic diversity for a northern high-latitude region. Diversity \&

276 Distributions 1-14 (2015).

277 29. Cardinale, B.J. et al. Biodiversity loss and its impact on humanity. Nature 486, 59-67

$278 \quad(2012)$.

279 30. Hoffmann, A.A. \& Sgro, C.M. Climate change and evolutionary adaptation. Nature 470,

$280 \quad 479-485(2011)$.

281

282 Supplementary Information is available in the online version of the paper

283

284

285 Correspondence to: Carlos E. Gonzalez-Orozco (cegonzalez@,corpoica.org.co ) or Bernd

286 Gruber (bernd.gruber@canberra.edu.au)

287 
289 We acknowledge the National Environmental Research Program (NERP) for workshop

290 funding, the Bjarne K Dahl Trust for support, and the ARC grant DP130101141 for

291 supporting the eucalypts phylogenetic work. BDM acknowledges a Visiting Fellowship from

292 the Collaborative Research Network for Murray-Darling Basin Futures, University of

293 Canberra in 2013. This manuscript includes work done by JTM while serving at the National

294 Science Foundation. The views expressed in this paper do not necessarily reflect those of the 295 National Science Foundation or the United States Government.

296

297

298

299

300

301

302

303

304

305

306

307

308

309

\section{Author contributions}

All authors contributed to project conception. C.G-O, L.J.P, A.H.T, N.K, B.G and S.W.L. conducted analyses. A.H.T, M.D, C.K and J.T.M developed the phylogeny. A.H.T, N.K, C.G-O, L.J.P and N.B compiled and corrected species spatial data records. C.G-O wrote the manuscript draft and all authors contributed to interpretation of the results and writing of the final paper.

02 


\section{Spatial Data and Species Distribution Modelling}

We modelled present and future distributions of current and four future time points (2025, 2045, 2065 and 2085$)$ for all 766 eucalypt species ( 163,000 occurrence records)

found in Australia (only 16 are endemic to another country) at a 5-km pixel resolution using a dispersal and a no-dispersal scenario. Data (presence-only) were extracted from Australia's Virtual Herbarium database [AVH] accessed in October 2011 (http://avh.chah.org.au/). one edaphic variable (median \% clay content derived from the 1:2 M Atlas of Australian 
modelled distributions to a more realistic spatial extent based on an initial trial of 20 species with good distribution data.

The AVH dataset is heavily influenced by road-based sampling, which was accounted for by including distance-to-roads as a predictor in each model, then setting distance-to-roads to zero for prediction (for present and future scenarios). Models were fitted using MaxEnt ${ }^{35}$ in the R package 'dismo' with a random background sample of 20,000 records (the same sample for each species), randomly distributed across Australia and uniform for all species.

We used only 'hinge features' because they tend to increase model performance without increasing model complexity ${ }^{36}$. Hinge features provide smoother response curves, which may more closely approximate the species' fundamental niche ${ }^{37}$. Model fit was evaluated with Area Under the ROC Curve from a 5-fold cross-validation for each species (see AUC values in SI 3). A Maxent logistic threshold (maximum training sensitivity plus specificity) for each species was applied to convert habitat suitability into presences and absences for present and future scenarios.

In the no-dispersal scenario, we used the same set of modelling parameters described above, but prevented colonization. The present distributions are identical in the dispersal and no-dispersal scenario. In each subsequent time period, species are not allowed to disperse to cells not occupied in the previous time step.

\section{Molecular data}

DNA data for the study was gathered by using existing and newly generated nuclear (ITS and ETS) and chloroplast sequences (matK and psba-trnH) that were successfully amplified for 711 eucalypt species (See Table in SI_5 for GenBank accessions). Leaves for DNA extraction were sourced from numerous field trips, botanical gardens, arboreta, and herbaria from multiple locations around Australia. In total 2560 gene sequences were used in the 
study, 237 were mined from GenBank, and 2323 were newly generated for this project. These sequences represented the 711 eucalypt species and 21 taxa from other Myrtaceae tribes which were used to root the phylogeny (Extended Data Fig. 6). Individual locus alignments were constructed using Muscle ${ }^{38}$ and adjusted manually in Se- $\mathrm{Al}^{39}$.

The phylogenetic analysis was performed on the concatenated alignment using maximum likelihood in the CIPRES Portal (www.phylo.org), utilising the RAxML HPC BlackBox tool with a partition model set for each locus, with bootstrapping set to automatically halt, which occurred after 550 replicates. The ML tree with bootstrap values is shown in Extended Data Fig. 7. Extended Data figure 8 is a bi-plot of branch length vs, bootstrap score, and shows that low bootstrap scores were restricted to very short branches. The uncertainty in these short branches did not affect the analyses presented here, since the better supported long branches contribute most of the PD.

\section{Species range shift analyses}

The mean centre of distributional area using the Maxent habitat suitability maps under present and future scenarios was estimated for the 657 species with matching phylogenetic data and SDMs following established methods ${ }^{40}$. This analysis showed the magnitude and direction of change of the mean centre of the climate space for each species between the present and 2085. Species were grouped according to their distribution change from the present, namely into "expanding" or "shrinking" if the predicted suitable climate space of a species increased or decreased between the present and 2085, respectively. Species whose suitable climate space was predicted to become extinct by 2085 were classified as "extinct". The 'dispersal' scenario assumes all species can disperse across the landscape. However, the use of spatial variables in the models indirectly accounts for some dispersal limitation, effectively limiting the potential of species to disperse to distant sites. We believe 
this spatial dispersal limitation is realistic given the biology of eucalypts, with most species having relatively heavy seeds without specialized dispersal mechanisms that simply drop to the ground when shed. Given the tree height, seed weights, and average wind speeds, most eucalypt species would not be expected to disperse beyond a single grid cell during the time period in this study without a rare long-distance dispersal event ${ }^{41}$.

\section{Spatial phylogenetic analyses of modelled distributions}

The SDM suitability values were aggregated at $\sim 25 \mathrm{~km} \times 25 \mathrm{~km}$ to a total of 12,813 grid cells and used to calculate Species Richness (SR), Weighted Endemism (WE), Phylogenetic Diversity (PD) ${ }^{10}$, Phylogenetic Endemism (PE) ${ }^{13}$, Relative Phylogenetic Diversity (RPD) and Relative Phylogenetic Endemism (RPE) in the Biodiverse software ${ }^{42}$ for 657 species. SR and WE results reported in Extended Data Fig. 1 were calculated on the basis of all 766 species because they did not require a phylogenetic tree. All phylogenetic metrics were calculated on the basis of the 657 species that are in the phylogeny. A spatial randomization based on 999 trials was applied to these metrics ${ }^{6}$. The purpose of the randomization is to identify whether the observed values were significantly different from a random selection of the same number of terminal taxa from the tree.

RPE is applied in a two-step test called categorical analysis of neo- and paleoendemism (CANAPE) using the same spatial randomization ${ }^{6}$. The first step of CANAPE determines whether a location is a center of high phylogenetic endemism by applying a onetailed test (for large values) to both the numerator and denominator of RPE (i.e., PE measured on the observed tree in the numerator and PE measured on a comparison tree where all branches are of equal (average) length in the denominator). Then, for grid cells passing this test, the second step applies a two-tailed test to the RPE ratio to identify cells dominated by unusually short range-restricted branches (centres of neo-endemism), unusually long range- 
restricted branches (centres of paleo-endemism), or both (centres of mixed endemism, termed

"super endemism" if both the numerator and denominator of RPE are highly significant) ${ }^{6}$.

\section{Phylogenetic turnover analyses of modelled distributions}

Two spatial turnover analyses using the Phylo Sørenson index ${ }^{43}$ were conducted in

Biodiverse $^{42}$ for 657 species to cross validate the observed patterns of the modelled PD distribution results: (1) a mean pair-wise rate of turnover between all pairs of cells within each time period, summarised using the mean and standard deviation for each year; and (2) the rate of phylogenetic turnover from the present to each modelled time period for each cell.

\section{References}

31. Euclid v3.0- Eucalypts of Australia (2002). https://www.anbg.gov.au/cpbr/cdkeys/euclid3/euclidsample/html/index.htm.

32. Kujala, H., Moilanen, A., Araújo, M.B. \& Cabeza, M. Conservation planning with uncertain climate change projections. Plos One 8, e53315 (2013).

33. Williams, K. J. et al. Harnessing continent-wide biodiversity datasets for prioritising national conservation investment. CSIRO Ecosystem Sciences, Canberra (A report prepared for the Department of Sustainability, Environment, Water, Population and Communities, Australian Government, Canberra) (2010).

34. McKenzie, N. J., Jacquier, D. W., Ashton, L. J \& Cresswell, H. P. Estimation of soil properties using the Atlas of Australian soils. CSIRO, Canberra (2000).

35. Elith, J. et al. A statistical explanation of Maxent for ecologists. Diversity and Distributions 17, 43-57 (2011).

36. Pollock, L.J. et al. Phylogenetic diversity meets conservation policy: small areas are key to preserving eucalypt lineages. Philosophical Transactions of the Royal Society B: Biological Sciences 370, 1662 (2015). 
37. Phillips, S.J \& Dudik, M. Modeling of species distributions with Maxent: new extensions and a comprehensive evaluation. Ecography 31, 161-175 (2008).

38. Edgar, R.C. MUSCLE: multiple sequence alignment with high accuracy and high throughput. Nucleic Acids Research 32, 1792-1797 (2004).

39. Rambaut, A. (2002) Sequence Alignment Editor (Se-Al) v2.0.

40. VanDerWal, J. et al. Focus on poleward shifts in species' distribution underestimates the fingerprint of climate change. Nature Climate Change 3, 239-243 (2013).

41. Cremer. Distance of seed dispersal in eucalypts estimated from seed weights. Australian Forest Research 7, 225-228 (1977).

42. Laffan, S.W., Lubarsky, E. \& Rosauer, D.F. Biodiverse, a tool for the spatial analysis of biological and related diversity. Ecography 33, 634-647 (2010).

43. Ferrier, S., Manion, G., Elith, J. \& Richardson, K. Using generalized dissimilarity modelling to analyse and predict patterns of beta diversity in regional biodiversity assessment. Diversity and Distributions 13, 252-264 (2007). 
$455 \quad$ Figure 1 | Variation in the predicted distribution of the suitable climate space of 657

456 Australian eucalypt species from 2014 to 2085. a, Shift in the predicted species

457 distribution where arrows present the magnitude and direction of change in distribution at the 458 centroid of the climatic space. $\mathbf{b}$, plots showing the general direction of the distributional 459 change for species that have shrinking distributions, expanding distributions or go extinct at 460 the last time point.

Figure 2 Areas of phylogenetic endemism using Categorical Analysis of Neo- And

Paleo-Endemism (CANAPE) for 657 species of eucalypts in Australia for projected

climate change scenarios at 2014 and 2085. a, CANAPE map for 2014, and b, 2085.

Ellipses indicate places where current areas of paleo-endemism disappear; rectangles indicate places where new areas of paleo-endemism appear; triangles indicate where current areas of paleo or neo-endemism weaken. Beige areas do not depart significantly from random expectation.

Figure 3 | Effects of projected climate change scenarios, between 2014 and 2085, on species of eucalypts found in current areas of paleo-endemism across Australia. Highlighted branches in the phylogenetic tree are present in the $\mathbf{7 3}$ grid cells that had significant paleo-endemism in Australia in 2014 (blue grid cells in ellipses Fig 2a).

472 Branches predicted to be lost from the 73 grid cells by 2085 are shown in blue, branches

473 predicted to immigrate by 2085 are shown in green, and branches common to both periods

474 are shown in red. Clades marked with an asterisk refer to places in the phylogeny

475 corresponding to paleo-endemism where individual branches were removed from the

476 phylogeny consistently over time. 


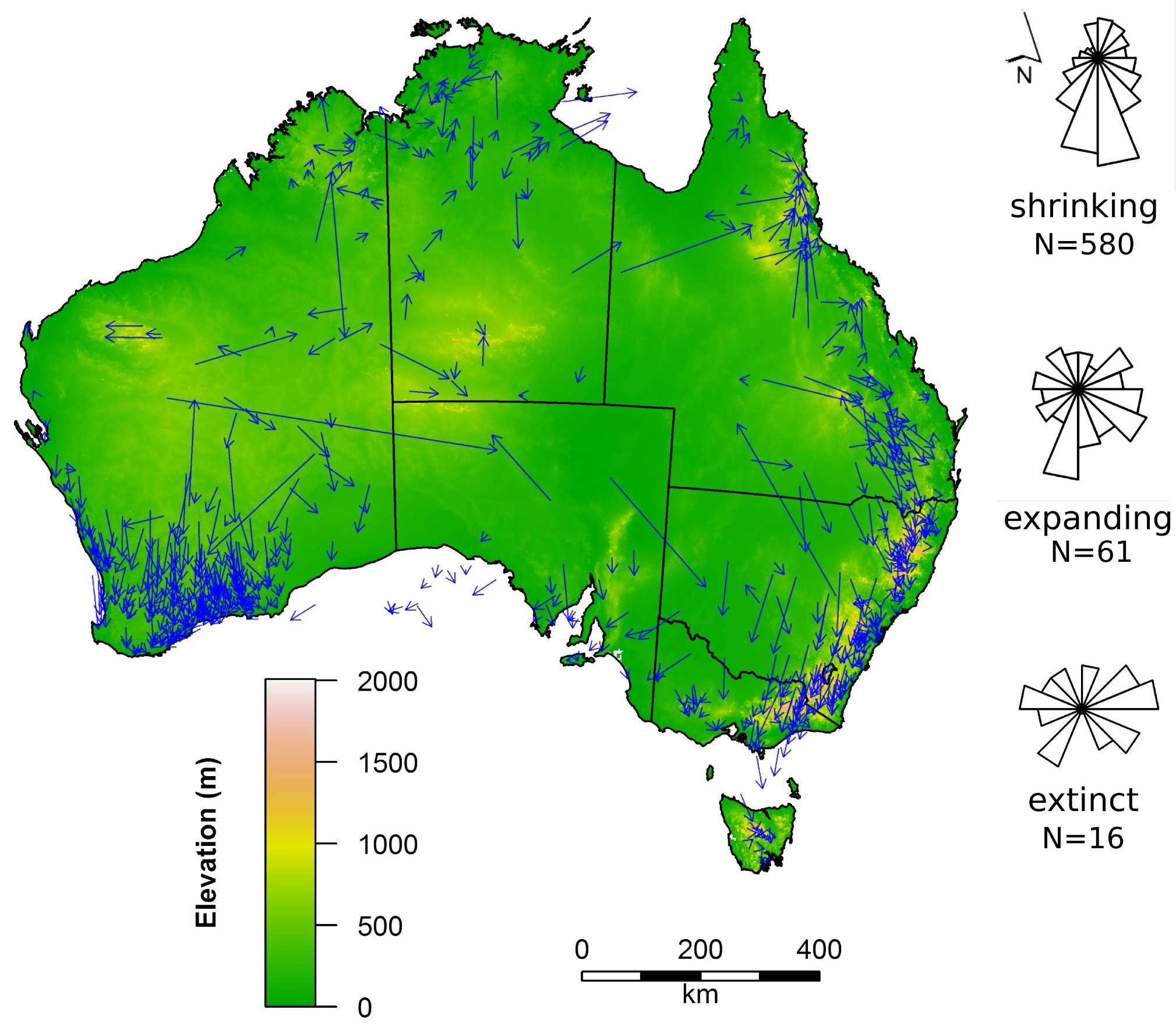


Categorical Analysis of Neo- And Paleo- Endemism a

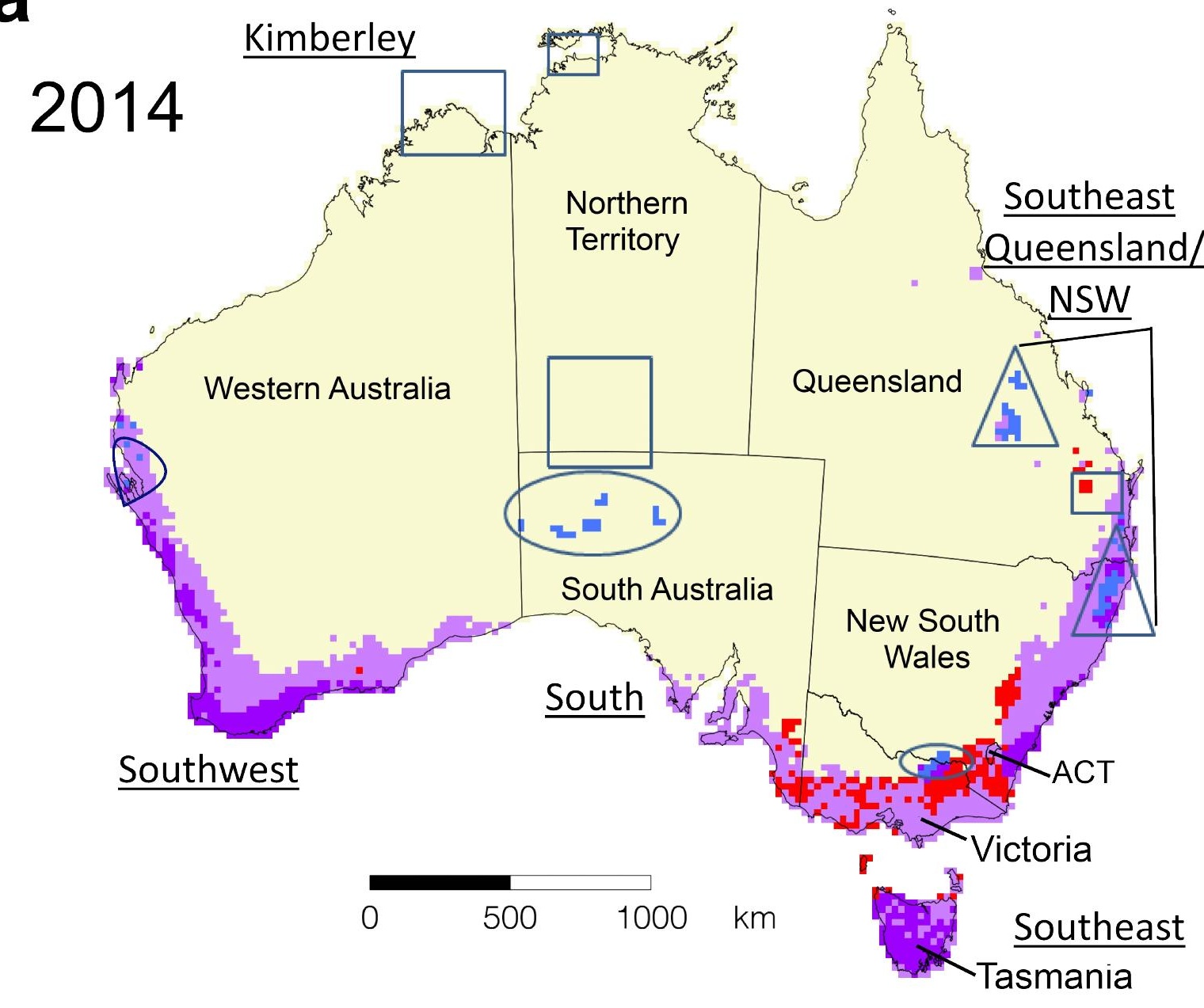

b

2085

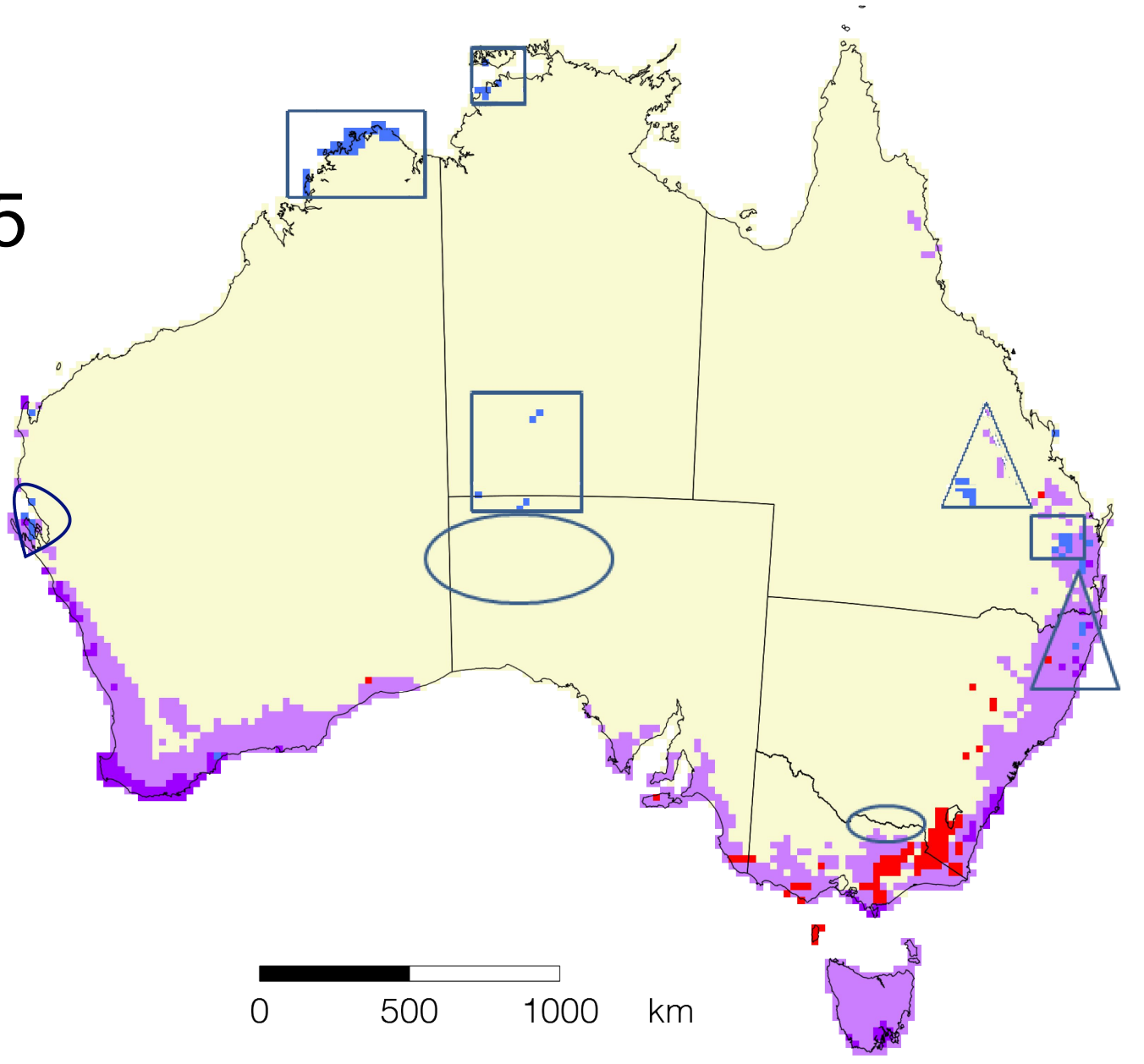

Neo Paleo

Mixed Super 
